\title{
Particles in Jupiter's atmosphere from the impacts of Comet P/Shoemaker-Levy 9
}

\author{
By ROBERT A. WEST \\ Jet Propulsion Lab, California Institute of Technology, 4800 Oak Grove Drive, Pasadena, CA \\ 91109, U.S.A.
}

\begin{abstract}
The dark clouds that were easily seen in small telescopes after the comet impacts were caused by small particles which were deposited in Jupiter's stratosphere. Observations from the Hubble Space Telescope and from ground-based instruments at visible and infrared wavelengths indicate that the mean radius of the particles is in the range 0.1 to $0.3 \mu \mathrm{m}$, and the total volume of particles is approximately the same as that for a 1-km diameter sphere. In the dark core regions of freshly-formed impacts, the particles are distributed over a large vertical extent, between about $1 \mathrm{mb}$ and $200 \mathrm{mb}$ or deeper. The diffuse outlying haze is confined to the high-altitude end of the range. Such a distribution probably reflects different methods of emplacement of the debris as a function of distance from the impact. The color of the particles, and their volatility as required to make waves visible, suggest an organic material as the main constituent. These relatively volatile materials are thought to have condensed onto more refractory grains after the plume material cooled, some 30 minutes or more after impact. The most refractory materials expected to condense from an evolving fireball are $\mathrm{Al}_{2} \mathrm{O}_{3}$, magnesium and iron silicates, and soot, depending on the $\mathrm{C} / \mathrm{O}$ ratio. A silicate spectral feature was observed, confirming that cometary material was incorporated into the grains, although silicate grains make up only 10 $20 \%$ of the particle volume. After one year in Jupiter's stratosphere, the particles have spread some $20^{\circ}$ in latitude and a significant number have sedimented into the troposphere where they are no longer visible.
\end{abstract}

\section{Introduction}

Impacts from fragments of comet Shoemaker-Levy 9 left visible marks on the jovian atmosphere, at wavelengths ranging from the ultraviolet to the near-infrared. The dark features (at visible wavelengths) were easily seen even in small telescopes, and were described by some observers as the most prominent markings ever seen on Jupiter. Clouds of small dust particles are largely responsible for these features.

Knowledge about the dust particles is of value for several reasons. They may give clues concerning the physics and chemistry of a high velocity impact into a deep atmosphere and subsequent fireball and plume eruption, with implications for the Earth. They constitute a considerable mass of observable material that may bear on questions surrounding the composition and chemistry of the impacting bodies and/or the chemistry of the jovian atmosphere at levels where we have no other information. Over longer time scales they serve as excellent tracers of wave motion and tracers of atmospheric mass motion in Jupiter's stratosphere where previously there were none. In this way they are analogous to sulfuric acid particles deposited in the Earth's stratosphere from volcanic eruptions, and their evolution during the first year after impact is in many ways similar to the evolution of terrestrial stratospheric particles.

This chapter will review the observations of the impact debris particles and address questions concerning their optical, physical and microphysical properties, composition, origin (whether from the comet or from Jupiter), and evolution. 


\section{Observations of impact debris particles}

Data relevant to aerosol studies fall into four main categories. These are (1) reflectivity and emissivity at ultraviolet (UV), visible, and infrared (IR) wavelengths, (2) observations of gas composition in the impact regions, (3) the visibility of waves, and (4) measurements made as a Galilean satellite moves into or out of eclipse (Mallama et al. 1995). The first of these categories contributes most directly toward studies of the debris particles. West et al. (1995) analyzed HST data at UV, visible and in the 889-nm methane and nearby continuum filters for a variety of spots both near the time of impact, and on a global scale up to a month after the last impact. Moreno et al. (1995) studied ground-based CCD images between 360 and $948 \mathrm{~nm}$ of impact $\mathrm{H}$ obtained at La Palma Observatory. Ortiz et al. (1995) reported on observations of images and spectra in the $\mathrm{K}$ and $\mathrm{H}$ bands $(1.5-2.4 \mu \mathrm{m})$ made at the Calar Alto Observatory. Banfield et al. (1995) obtained images in the spectral range $2.0-2.3 \mu \mathrm{m}$ taken on the $5 \mathrm{~m}$ Hale telescope at Palomar mountain. Orton et al. $(1995 \mathrm{a} ; 1995 \mathrm{~b})$ obtained images at several wavelengths in the same spectral region, and in the very strong $3.4-\mu$ m methane band as well from the NASA Infrared Telescope Facility. Nicholson et al. (1995) provided spectra of the emerging plume from the $\mathrm{R}$ impact in the thermal IR $(8 \mu \mathrm{m}$ to $13 \mu \mathrm{m})$ observed from Mt. Palomar. Rosenqvist et al. (1995) obtained spectra near $4 \mu \mathrm{m}$ of the area around the W and $\mathrm{K}$ impact sites with the $6 \mathrm{~m}$ telescope of the Special Astronomical Observatory of Zelenchuk.

\section{Particle optical properties, optical depth, mean radius, and total volume}

At wavelengths outside the strong methane absorption bands, Particles appear as dark markings over a very broad spectral range, from the ultraviolet to $1 \mu \mathrm{m}$ in the nearinfrared. Signatures of particles are seen in Hubble Space Telescope (hereafter HST) UV images (Clarke et al. 1995; West et. al. 1995) and spectra at wavelengths as short as $125 \mathrm{~nm}$ (Noll et al. 1995). Figure 1 shows the appearance of many fresh impact sites on 21 July, 1994 in the HST Wide Field/Planetary Camera 2 (hereafter WFPC2) imaged with the F255W filter which has a solar flux-weighted effective wavelength of $275 \mathrm{~nm}$.

The darkest region of the planet in Fig. 1 is the cloud from the $\mathrm{H}$ impact near the dawn terminator. It was immediately obvious from these images that the particles reside at high altitudes in Jupiter's stratosphere, otherwise scattering from the overlying gas particles would make the limb brighter than it is. At the nadir $275-\mathrm{nm}$ photons penetrate to 0.35 bar by the time scattering by $\mathrm{H}_{2}$ and He attenuates the beam by the factor $1 / e$. Near the limb where the slant path is large, the pressure level sampled by $275-\mathrm{nm}$ photons is just a few mb.

The debris particles have a flat or slightly brown spectrum at visible wavelengths, out to $1 \mu \mathrm{m}$. Figure 2 is a half-tone version of a color composite made from HST WFPC2 images in the blue (F410M, effective wavelength $410 \mathrm{~nm}$ ), green (F555W, $555 \mathrm{~nm}$ ), and near-IR (F953N, $955 \mathrm{~nm})$. The image was taken almost 2 hours after the impact of fragment G. The impact from fragment D is also seen in Fig. 2. Several features are to be noted. The main features are (1) dark core regions, (2) circular rings, and (3) a crescent-shaped region at larger distance from the core. A key observation, evident in Fig. 2, is that the color of the particles in all of these regions is the same.

The lowest reflectivities occur at the center of the impact. These core regions have a central circular shape, about $2000 \mathrm{~km}$ in diameter for $\mathrm{G}$, with a wedge of material fanning out to the southeast. 


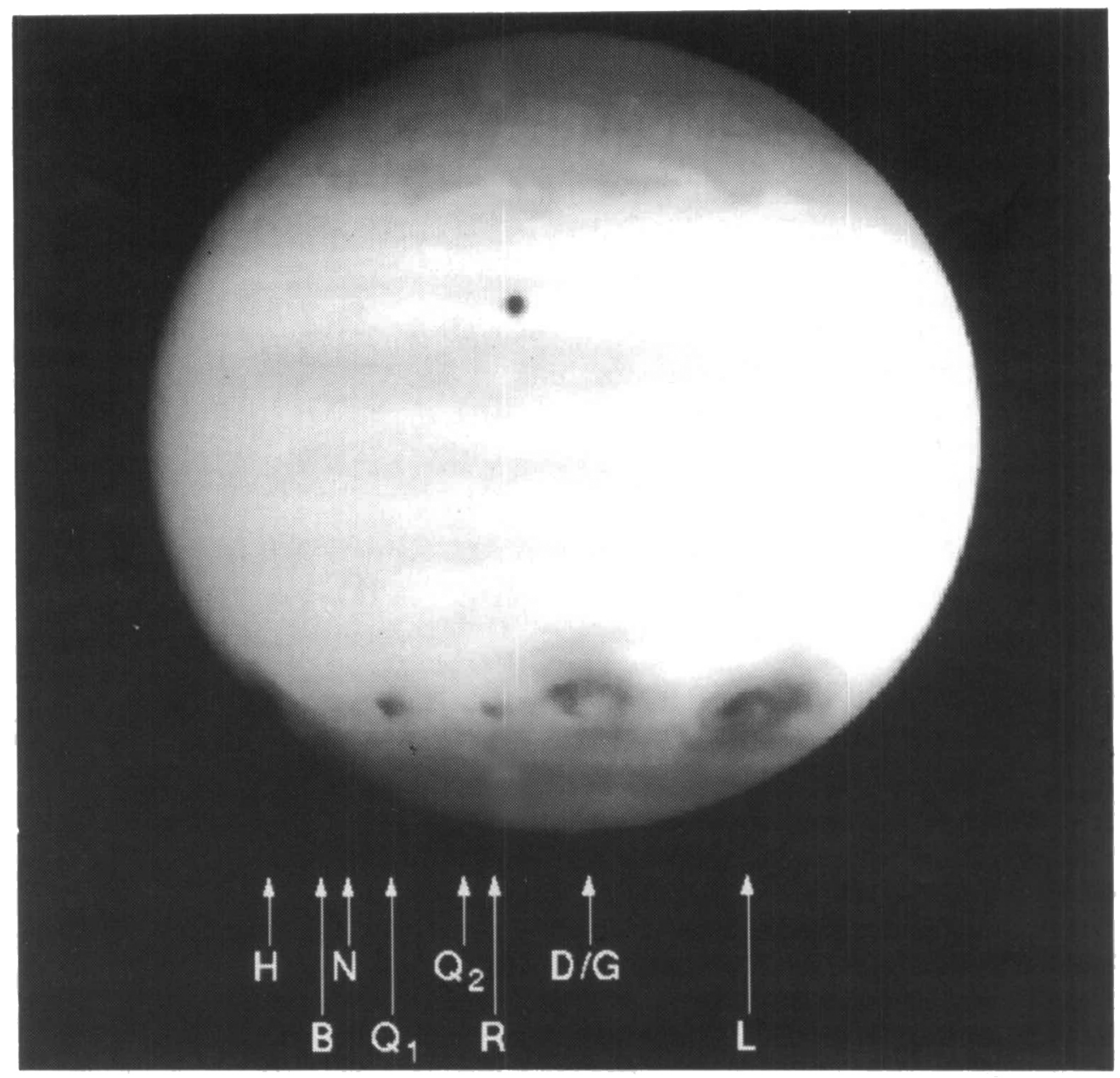

Figure 1. Ultraviolet image of Jupiter taken by the Wide Field Camera 2 on NASA's Hubble Space Telescope. The image shows Jupiter's atmosphere at a wavelength of $275 \mathrm{~nm}$ after many impacts by fragments of comet Shoemaker-Levy 9 . The most recent impactor is fragment $R$. This photo was taken on 21 July 1994, about 2.5 hours after the $R$ impact. A large, dark patch from the impact of fragment $\mathrm{H}$ is visible rising on the morning (left) side. Jupiter's moon Io is the dark spot just above the center of the planet.

A thin circular ring in Fig. 2 is centered on the core region and is observed to expand radially at a rate of about $450 \mathrm{~km} \mathrm{~s}^{-1}$ (Hammel et al. 1995; Ingersoll and Kanamori, 1995). Similar rings were seen in HST images of impacts A, E, Q1 and R. These features are also visible as bright rings in the $890-\mathrm{nm}$ methane-band images, proving that they are made visible by the presence of particles in Jupiter's stratosphere. Inside of this ring is a fainter one whose expansion velocity is roughly half that of the more visible ring (Hammel et al. 1995, Ingersoll and Kanamori, 1995). Ingersoll and Kanamori argued that the wave speed is determined by the lapse rate in the water cloud region between 6 and 10 bars pressure, but temperature perturbations in the stratosphere are responsible for particle formation during the passage of a wave (see the chapter by Ingersoll and Kanamori in this volume). This mechanism for particle formation provides a constraint on the vapor pressure of the condensing material and hence its composition. 
Figure 2. The $\mathrm{D}$ and $\mathrm{G}$ impact sites as they appeared almost 2 hours after the $\mathrm{G}$ impact (from West et al. 1995). The diameter of the wave (circular ring centered on the G impact) is about $7000 \mathrm{~km}$. A color version of this figure appears in West et al. (1995). 


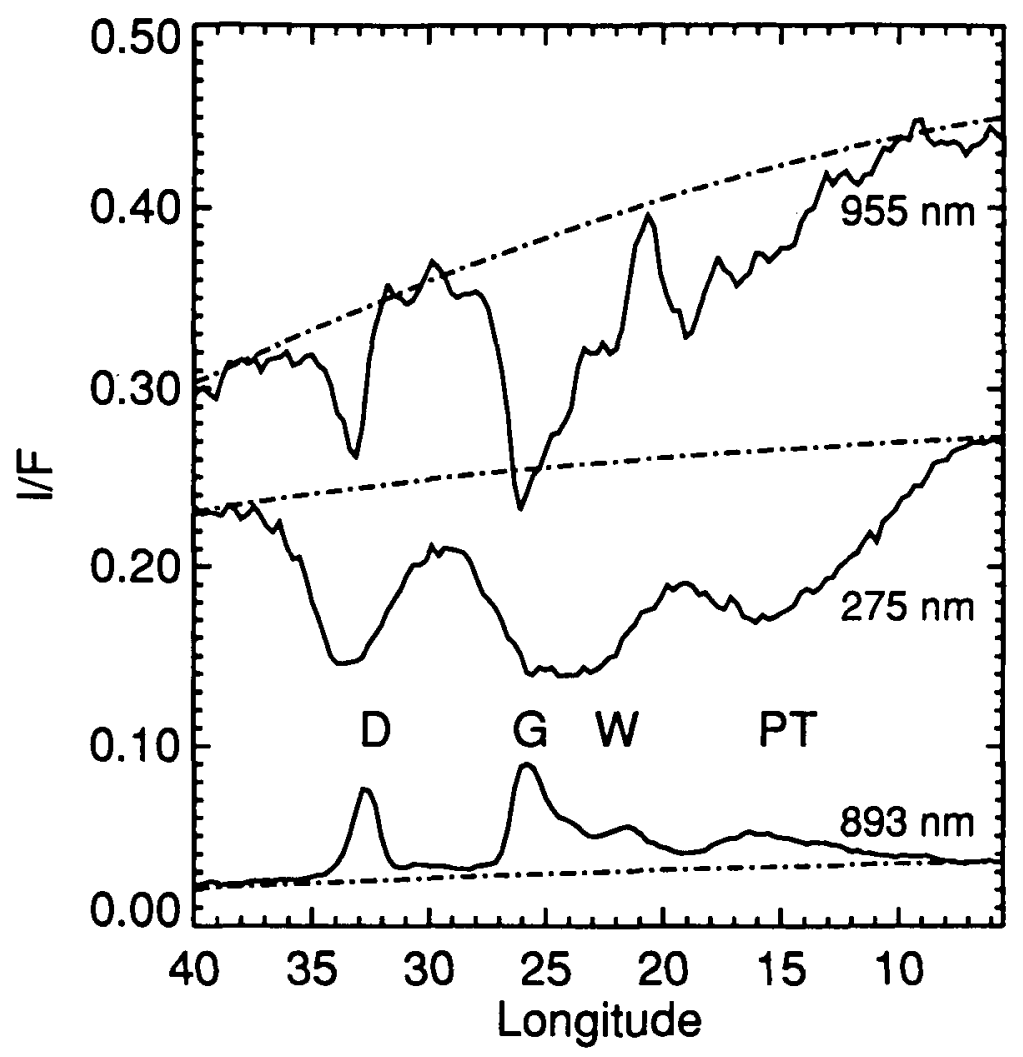

FIGURE 3. The ratio of intensity, I, observed by the WFPC2 to incident solar flux $(\pi \mathrm{F})$ is shown for a cut across the cores of the D and G impact sites shown in Fig. 2, along a line of constant latitude at three wavelengths (from West et al. 1995). The dot-dash lines indicate the level of the longitudinal-average brightness of the undisturbed adjacent regions determined by fitting adjacent regions to a generalized Minnaert law: $\ln (\mu \mathrm{I} / \mathrm{F})=\mathrm{C}_{0}+\mathrm{C}_{1} \mathrm{x}+\mathrm{C}_{1} \mathrm{x}^{2}+\mathrm{C}_{1} \mathrm{x}^{3}$, where $\mathrm{x}=$ $\ln \left(\mu \mu_{0}\right)$ and $\mu$ and $\mu_{0}$ are the cosines of the emission angle and solar incidence angle, respectively. The $\mathrm{C}$ coefficients were obtained by a linear least-squares singular value decomposion algorithm. The dark cores of the $D$ and $G$ sites are indicated, along with the location of the wave (W) and plume terminus (PT) or optically thin crescent (from West et al. 1995).

The crescent of dark material lying at greater distance from the core is optically thin at visible wavelengths. Contrast from features in the troposphere can be seen through the haze. The location of the haze is consistent with the idea that particles in the outlying regions were initially ejected at large velocity from an erupting plume seen on the limb in HST and some high-resolution ground-based images (see Hammel et al. 1995 and the chapter by Hammel in this volume).

The magnitude of the contrast can be judged quantitatively from Fig. 3. The features are more diffuse at $275 \mathrm{~nm}$ compared to longer wavelength both because the optically thin regions are optically thicker at UV wavelengths, and because there is some image smear in the UV due to the long exposure time. In addition, some of the absorption in the F255W filter is due to $\mathrm{S}_{2}$ gas (Noll et al. 1995). The $893 \mathrm{~nm}$ filter is centered on a methane band where the ambient atmosphere value for $\mathrm{I} / \mathrm{F}$ is close to 0.015 at the geometry of the data plotted in Fig. 3. The much higher $\mathrm{I} / \mathrm{F}$ in the cores of the spots is due to significant aerosol optical depth in the stratosphere. The particles are darker than the ambient atmosphere in the nearby $955 \mathrm{~nm}$ continuum band where methane 


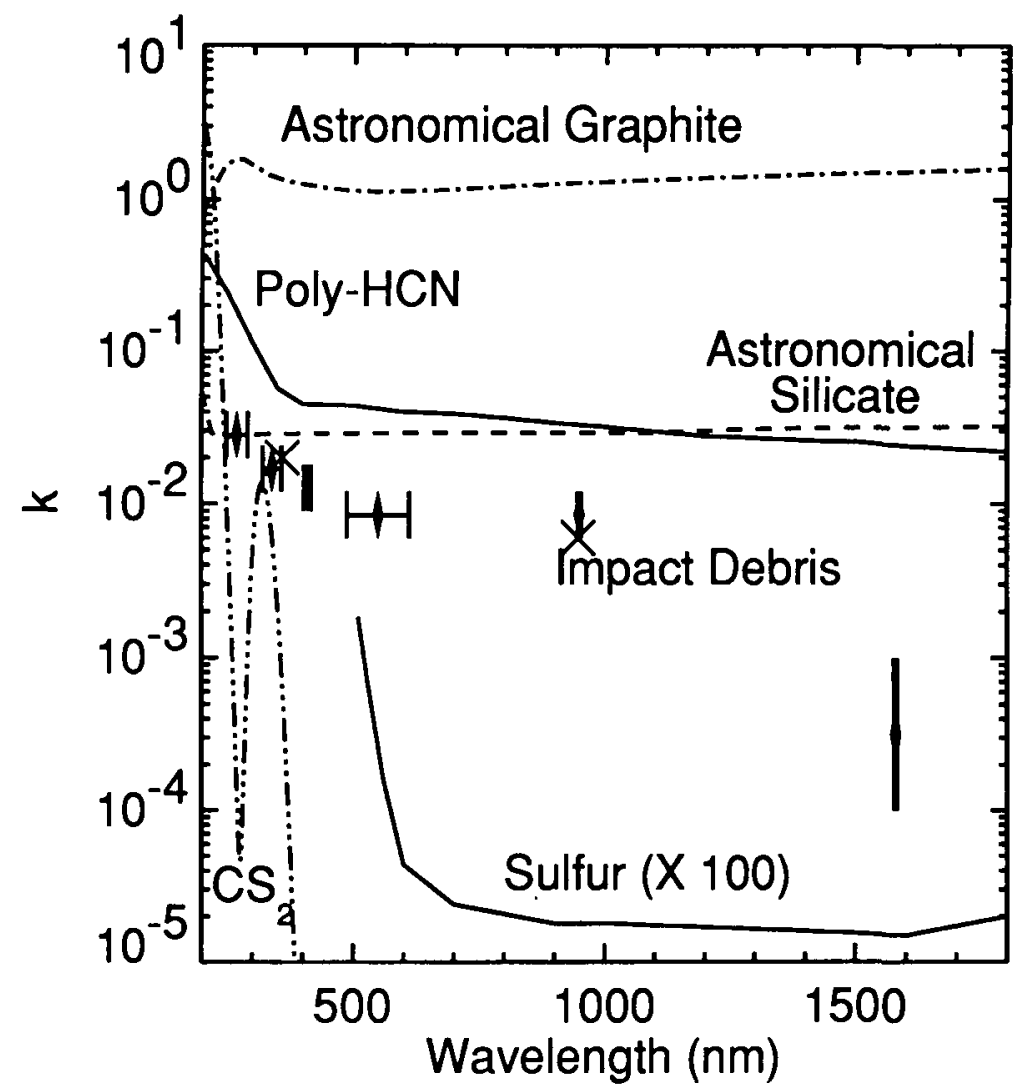

FIGURE 4. The imaginary part of the refractive index $(k)$ derived at several wavelengths below $1 \mu \mathrm{m}$ from HST images (West et al. 1995, diamonds with error bars) and ground-based images (Moreno et al. 1995, $\times$ symbols), and at $1.58 \mu \mathrm{m}$ by Ortiz et al. 1995. The results derived by West et al. include bars showing the HST WFPC2 filter passbands and uncertainty in k. Also shown are values for several candidate materials (from Gustavsen, 1989; Sasson et al. 1985; Draine, 1985; Khare et al. 1994).

absorption is small. Moreno et, al. (1995) found results from ground-based CCD images similar to those reported by West et al. (1995).

At longer wavelengths the contrast at continuum wavelengths is much reduced. Ortiz et al. (1995) reported difficulty detecting the spots in the $1.58 \mu \mathrm{m}$ continuum band, indicating that the optical depth is smaller or the imaginary refractive index is smaller than at shorter wavelengths, or a combination of the two.

West et al. (1995) studied the darkest core regions of several of the fresh impact sites where the large optical depth greatly reduced contributions from the underlying atmosphere to the observed intensities. Multiple scattering models for the reflectivity and optical depth of the particles in these regions provided estimates of the imaginary part of the refractive index of the particles as well as their mean radius. Ortiz et al. (1995) followed a similar procedure for their near-infrared spectra of the G/D complex and the $\mathrm{H}$ impact site. Results are shown in Figure 4.

Mie theory for spheres was used in both studies to relate refractive index to particle optical properties. Since no observations exist which could differentiate between spherical and nonspherical particles we do not know if Mie theory is appropriate. It is quite possible that the particles are clusters of smaller monomers, as proposed for the jovian polar stratospheric aerosols (West and Smith, 1991). If that is the case the value of $k$ 
derived using Mie theory, as well as the particle size, could be in error. West (1991) compared optical properties of small aggregate particles to spheres.

Even if the assumption of spherical shape is correct, the particle size distribution and the real part of the refractive index contribute to the optical properties and to the uncertainty in the derived value of $k$. There is not enough information to determine the particle size distribution. The first moment of the size distribution (the particle mean radius) can be estimated from the wavelength dependence of the optical depth. The most thorough studies to date are those by West et al. Moreno et al. (1995), and Ortiz et al. (1995), covering the spectral range from $275 \mathrm{~nm}$ to $1.58 \mu \mathrm{m}$. Rosenquist et al. (1985) derived average optical depth at $3.9 \mu \mathrm{m}$ for a 3 -arcsec region centered on the $\mathrm{K}$ and $\mathrm{W}$ impact sites. They found optical depths in the range $0.07-0.15$ during the first few days after the impacts.

An estimation of particle mean radius requires knowledge or an assumption of the real part of the refractive index. West et al. (1995) assumed the real part of the refractive index to be 1.4-1.44, consistent with many organic and some inorganic $\left(\mathrm{NH}_{3}\right.$ ice) compounds. West et al. were able to fit the observed I/F provided particle mean radii were in the range 0.15 to $0.3 \mu \mathrm{m}$, with the imaginary part of the refractive index also uncertain by a factor of 2 as shown in Fig. 4. The uncertainty in $\mathrm{k}$ is larger at $1.58 \mu \mathrm{m}$ because both the optical depth and the contrast decrease at the longer wavelength. Ortiz et al. (1995) and Moreno et al. (1995) assumed the real part of the refractive index to be 1.7 and found a value of $0.15 \mu \mathrm{m}$ for the particle radius, on the low end of the range determined by West et al. Mie calculations are sensitive to the product (n-1)a/ $\lambda$ where $\mathrm{n}$ is the real part of the refractive index, $\mathrm{a}$ is the particle radius, and $\lambda$ is the wavelength. The results for particle mean radius derived by Ortiz et al. and Moreno et al. are near the middle of the range derived by West et al. when scaled by the factor (n-1). Although the optically thick core regions were used to determine refractive index, finite optical depth and uncertainty in the vertical location of the haze particles also contribute to uncertainty in optical depth, especially in the infrared. Additional modeling is needed to better define the values and their uncertainties.

Aerosol optical depth generated by the impacts depends strongly on location, time and wavelength. Near the optically thick core regions of a fresh impact, the contrast between the absorbing material and the ambient cloud reflectivity can change by more than a factor of 2 over a distance of $1000 \mathrm{~km}$. The best ground-based images (atmospheric blur disk $\sim 0.4$ arcsec) can resolve features as small as $1500 \mathrm{~km}$. The HST Planetary Camera-2 was able to resolve (at the Nyquist frequency) features as small as $342 \mathrm{~km}$. West et al. (1995) and Moreno et al. (1995) found 889-nm optical depths near 4 for the core regions of several fresh impacts. Optical depth at other wavelengths can be estimated from Fig. 5 which shows the wavelength dependence of the extinction cross section for size distributions of spheres. The ratio of optical depth between $950 \mathrm{~nm}$ and $275 \mathrm{~nm}$ changes by a factor of 3 when the particle radius changes only by a factor of 1.4 from 0.21 to $0.28 \mu \mathrm{m}$.

West et al. (1995) generated maps of the optical depth of the particles at two wavelengths (275 and $889 \mathrm{~nm}$ ), shown in Fig. 6. The largest optical depths are greater than 12 at $275 \mathrm{~nm}$ one day after the last of the impacts. During the following week and month the material diffused horizontally due to the motion of vortices and the zonal wind shear. The role of small vortices is especially apparent on $23 \mathrm{July}$. The arcs of material extending to the north and south of impacts $A$ and $E$ near longitudes $180^{\circ}$ and $150^{\circ}$, respectively appear to be following the flow pattern of nearby vortices (see the chapter by Beebe). 


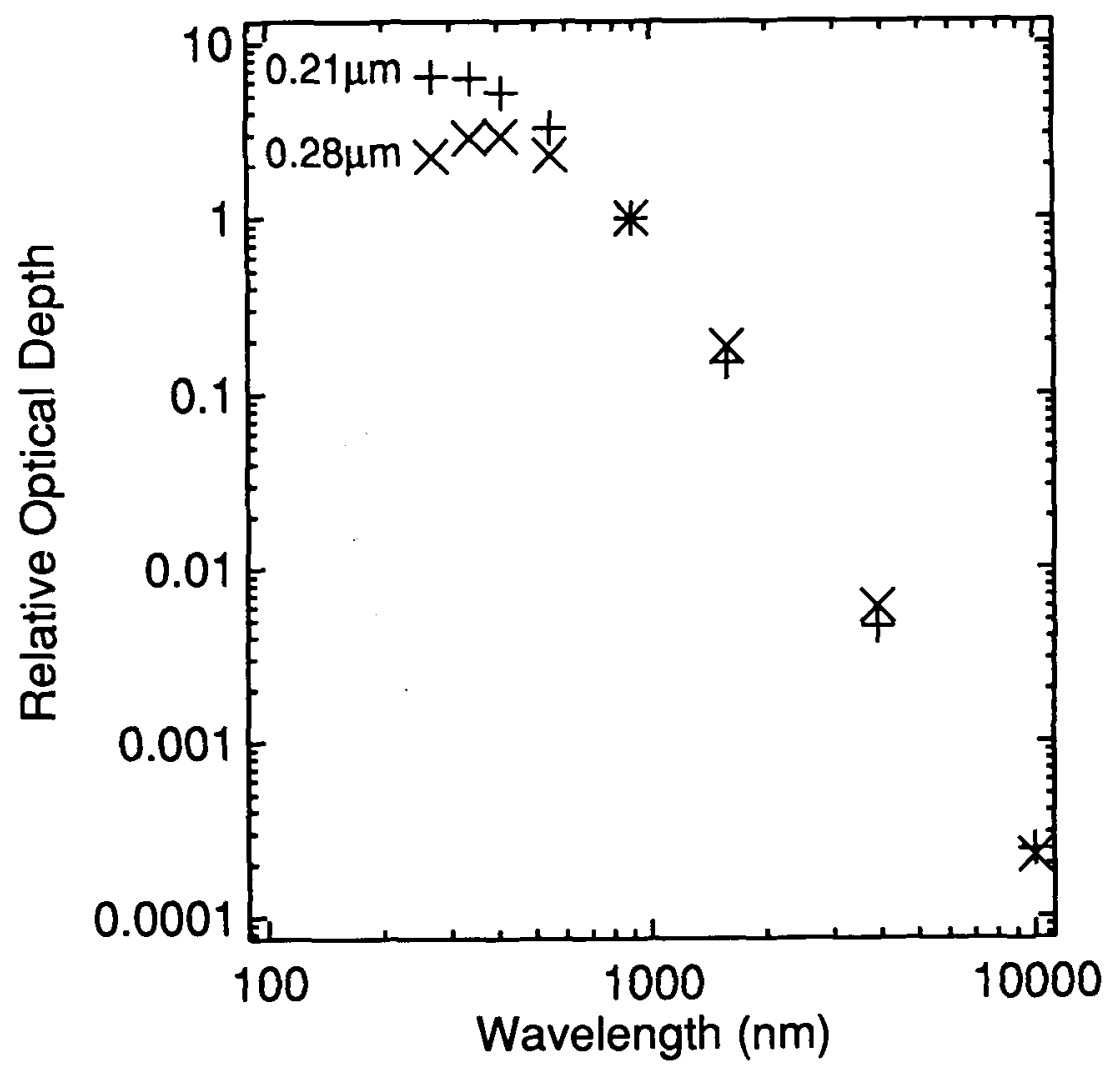

FiguRE 5. Wavelength dependence of the optical depth for particles whose mean radius is 0.21 and $0.28 \mu \mathrm{m}$, as indicated. Both curves are normalized to $950 \mathrm{~nm}$ and both were calculated with Mie theory for a real refractive index of 1.44 and for an imaginary part that varies as shown in Fig. 4 for the impact debris. The imaginary refractive index at 3.9 and $10 \mu \mathrm{m}$ has the same value $\left(3 \times 10^{-4}\right)$ as that used for the point at $1.58 \mu \mathrm{m}$. The particle emissivity at the longer wavelengths is proportional to the imaginary part of the polarizibility of the material which is related to the imaginary refractive index. Values for the optical constants of the debris particles at wavelengths longer $1.58 \mu \mathrm{m}$ are not known.

West et. al. integrated the particle optical depth over 360 degrees of longitude and 17.7 degrees of latitude corresponding to the latitude bands in Fig. 6. The total area of that region is $6.48 \times 10^{9} \mathrm{~km}^{2}$. The total integrated optical depth on $23 \mathrm{July,} 30 \mathrm{July}$, and 24 August was $7.0,4.6$, and 3.0 (all $10^{9} \mathrm{~km}^{2}$ ) at $275 \mathrm{~nm}$. The optical depth at $893 \mathrm{~nm}$ on the same dates was $1.1,1.5$, and $1.5 \times 10^{9} \mathrm{~km}^{2}$. The ratio of optical depth at the two wavelengths on the same three dates is 6.4, 3.1, and 2.0. As seen in Fig. 5, the ratio on 23 July implies the particle mean radius was $0.21 \mu \mathrm{m}$, while on $30 \mathrm{July}$ and August it was $0.24 \mu \mathrm{m}$ and $0.28 \mu \mathrm{m}$. West et al. argued that coagulation of particles could account for an increase in particle mean radius during the month following the impacts.

The total volume of the debris particles can be calculated from knowledge of the particle mean radius, refractive index, and optical depth. Using the values of refractive index assumed $(\mathrm{n}=1.44)$ and derived (Fig. 4), West et al. (1995) calculated optical cross sections of particles with the mean radii mentioned above. The total number of particles is given (assuming all particles have the mean radius) by the total optical depth divided by the extinction cross section of an individual particle. The total volume is then the total number of particles times the volume of each particle. West et al. found total 


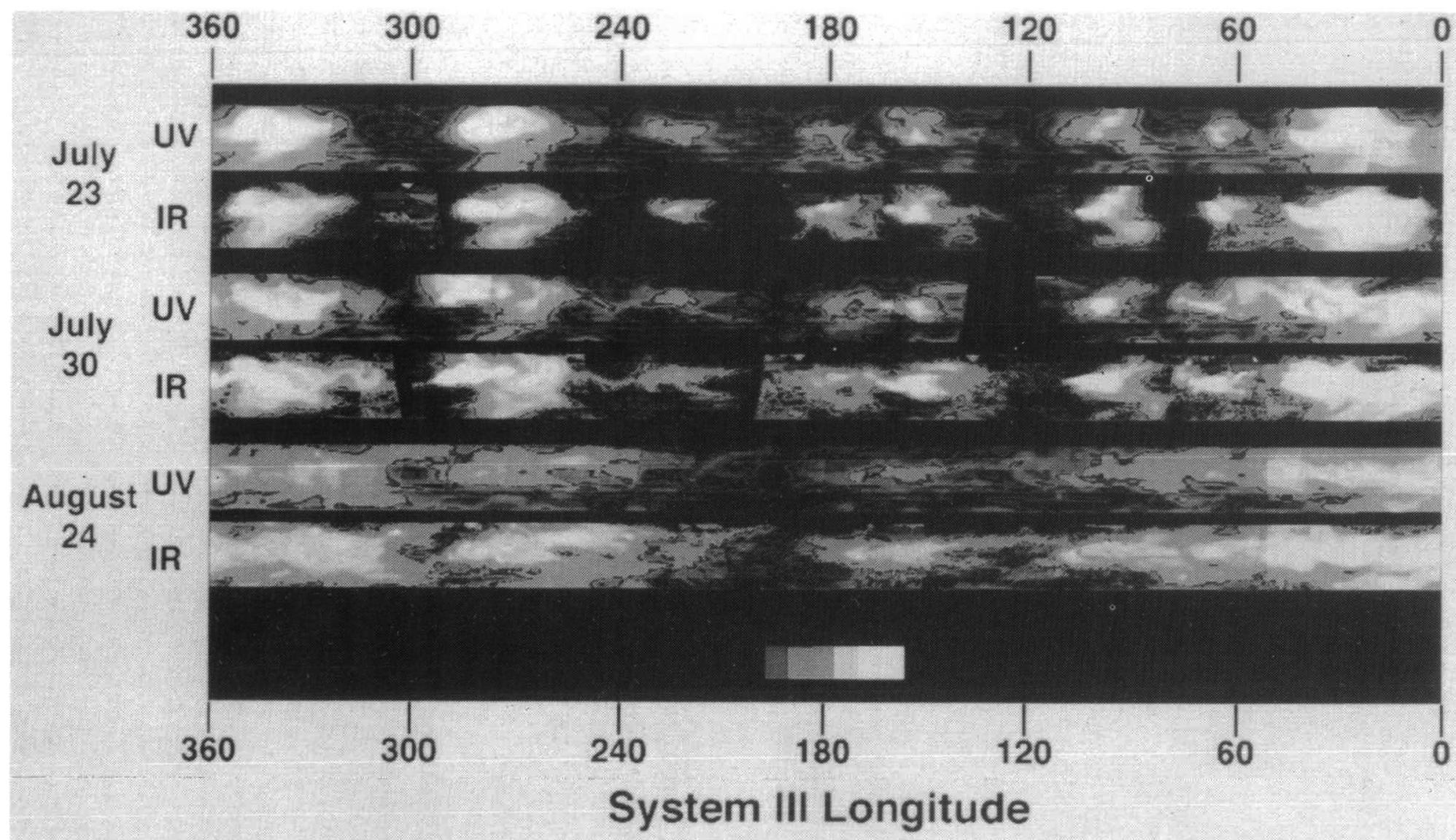

Figure 6. Optical depths of debris material are shown for wavelengths $275 \mathrm{~nm}$ and $893 \mathrm{~nm}$ as functions of latitude (between $36.5^{\circ} \mathrm{S}$ and $54.2^{\circ} \mathrm{S}$ ) and longitude for $23 \mathrm{July,} 30 \mathrm{July}$, and 24 August (from West et al. 1995). Each strip has latitude $36.5^{\circ} \mathrm{S}$ at the top and latitude $54.2^{\circ} \mathrm{S}$ at the bottom. The intensity steps at the bottom calibrate the colors in terms of the optical depth. The brightest intensities correspond to optical depth 12 or higher, and the lowest correspond to optical depth 0.2 (adapted from a color figure by West et al. 1995). 
particle volume to be equal to the volume of a sphere of radius $0.51,0.52$, and $0.45 \mathrm{~km}$ on the three dates. The particles at the large end of the size distribution probably account for the apparent loss of about $30 \%$ of the particle volume between 23 July and 24 August, augmented by horizontal eddy diffusion (from vortices as discussed previously) of particles outside the latitude strip studied by West et al. Banfield et al. (1995) analyzed images of Jupiter in the wavelength range $2.0-2.35 \mu \mathrm{m}$ taken on the $5 \mathrm{~m}$ Hale telescope at Palomar mountain. They derived total particle volume to be equal to that of spheres with radii $0.67,0.60$ and $0.65 \mathrm{~km}$ in late July, mid-August, and late August of 1995 . Those values are close to the values derived by West et al. given the uncertainties in both studies. The somewhat larger volumes derived by Banfield et al. may be due to the greater latitude coverage of that study compared to West et al. who retrieved optical depth only between latitudes $36.5^{\circ} \mathrm{S}$ and $54.2^{\circ} \mathrm{S}$.

Moreno et, al. (1995) estimated the volume of particulate material deposited by the $\mathrm{H}$ impact from an analysis similar to that done by West et al. (1995). They found a value of $115 \mathrm{~m}$ for the radius of an equal-volume sphere. That value is 80 times smaller than the volume for the globally integrated debris found by West et al., even though the $\mathrm{H}$ impact probably contributed more than $5 \%$ of the total optical depth. Recalling that the Mie results depend on the product $(n-1) \times a$, and that Moreno et al. used $n=1.7$ and $\mathrm{a}=0.15 \mu \mathrm{m}$, whereas West et al. used $\mathrm{n}=1.44$ and $\mathrm{a}=0.25 \mu \mathrm{m}$, the factor of roughly 4 in derived volume difference can be attributed to the different trades between particle mean radius and the real part of the refractive index. Since the real part of the refractive index is not known, its uncertainty leads to a factor of 4 or more uncertainty in particle volume, with larger volumes derived under assumption of smaller real refractive index.

\section{Particle Composition}

The solid particles formed by the impacts constitute a substantial fraction of the total debris, and a determination of their composition would be valuable, with implications for the composition of the impacting body and Jupiter's atmosphere. Clues to composition come from (1) the wavelength dependence and absolute value of the imaginary refractive index of the particles, (2) observations of gas-phase constituents such as water vapor which would condense after the initial hot phase of the fireball and fallback, and other constituents such as $\mathrm{HCN}$ and $\mathrm{CS}_{2}$ which coexist with the solid, and (3) the detection of particles formed by waves which constrains the combination of mixing ratio and vapor pressure of the material in the phase transition region (the cooling phase of the wave). Chapters in this volume by Lellouch, Moses, and Ingersoll and Kanamori are devoted to the gas-phase composition and the physics of the impact-generated waves. Only those aspects which touch upon the composition of the particulates will be treated here.

At least some of the material is of cometary origin. Emission from metal and silicate atoms and ions were observed shortly after some of the impacts. Noll et al. (1995) reported on ultraviolet spectral features of iron, silicon, and magnesium. Roos-Serote et al. (1995) observed emission lines from sodium, iron, calcium, lithium and potassium. The emission lines were observed soon after impact and were not observed afterward. The atoms responsible for these emissions reside at very low pressures and possibly high $(\sim 1000 \mathrm{~K})$ temperatures. Their relation to the particulate matter is unclear.

An observation of greater significance to aerosol composition was made by Nicholson et al. (1995) who obtained spectra in the region between 8 and $13 \mu \mathrm{m}$ (Fig. 7). The measurements were made as the plume material was falling back into the atmosphere and the thermal energy generated from the process heated the gas and particles. Nicholson 


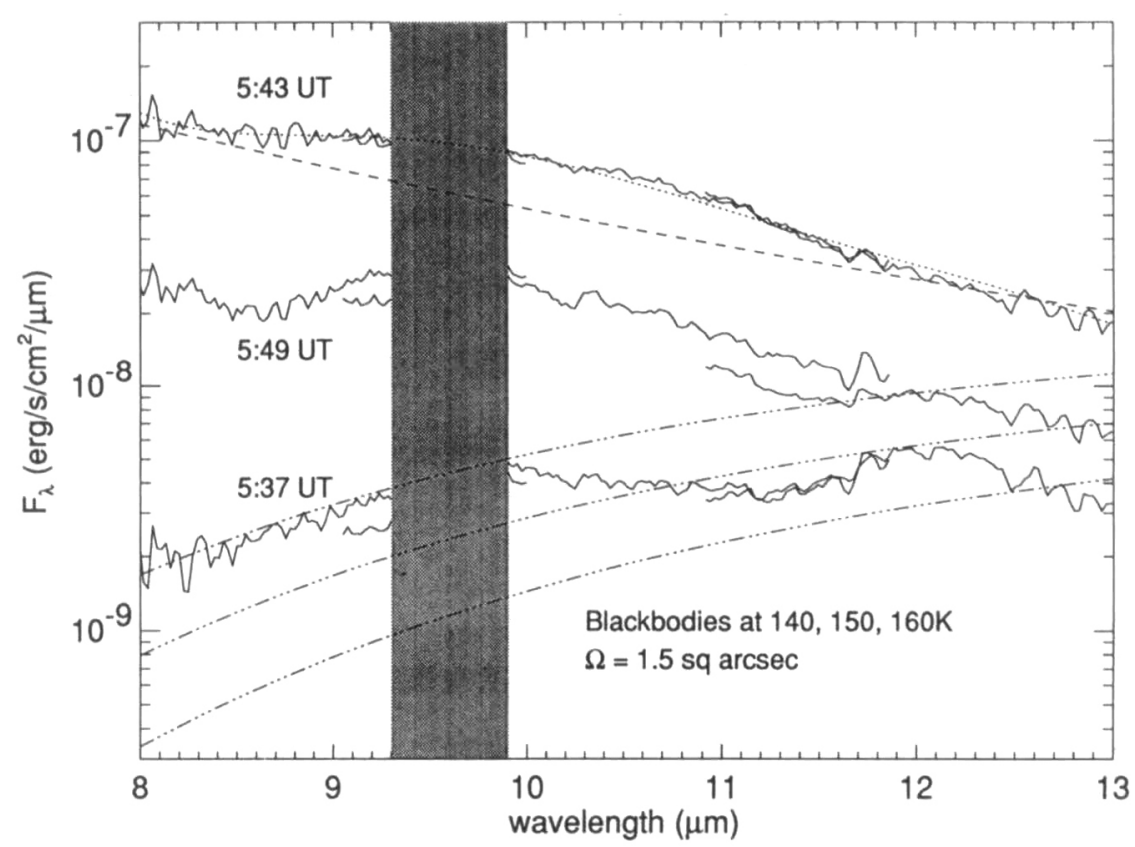

Figure 7. (From Nicholson et al. 1995) Spectra (flux) observed during the R impact on 21 July, 1995 from Palomar Observatory. The first spectrum (5:37 UT) was obtained prior to significant brightening at $10 \mu \mathrm{m}$. The second (5:43 UT) was obtained just prior to peak brightness, and the third (5:49 UT) during the decay phase. The dashed curve shows flux calculated for a $2000 \mathrm{~K}$ blackbody. The dotted curve which tracks the data at 5:43 UT is a synthetic spectrum using optical constants for "astronomical silicate" (Draine, 1985). Dot-dash curves show blackbody fluxes corresponding 140,150, and $160 \mathrm{~K}$. The stippled region between 9 and $10 \mu \mathrm{m}$ was obscured by telluric opacity.

et al. assumed emission from methane gas could be modeled by a power-law function of wavelength. They used the data to find the best value of the exponent $(-7.5)$, along with a temperature $(370 \mathrm{~K})$ and the product of the effective solid angle of the emitting dust and its optical depth at $9.7 \mu \mathrm{m}$. The fit to the spectrum is shown in Fig. 7 .

Nicholson et al. derived a value of $6 \times 10^{12} \mathrm{~g}$ for the total mass of silicate dust generated by the $\mathrm{R}$ impact. According to Banfield et al. (1995), the $\mathrm{R}$ impact contributed only $2 \%$ of the total number of particles to the globally integrated debris. Therefore the total mass of silicate material could be $3 \times 10^{14} \mathrm{~g}$, and its volume (assuming a density of $3.3 \mathrm{~g} \mathrm{~cm}^{-3}$ ) equal to that for a sphere of radius $0.28 \mathrm{~km}$, some $17 \%$ of the total particulate volume derived by West et al. (1995), and $10 \%$ of the total volume derived by Banfield et al. Such a large proportion of silicate material would be consistent with the idea that a large fraction, perhaps most, of the ejected material was of cometary origin.

Water, either from the impacting body or from Jupiter, was expected to contribute to the debris inventory, and water vapor lines were observed by ground-based observers (Meadows and Crisp, 1995) and observers on the Kuiper Airborne Observatory (Bjoraker et al. 1995). Water lines were observed only when the plume was visible and when temperatures were warm ( $1000 \mathrm{~K}$ or more according to Bjoraker et al.). Bjoraker et al. report masses of water for the $\mathrm{G}$ and $\mathrm{K}$ impacts to be each in the range 1.4 to $2.8 \times 10^{12} \mathrm{~g}$, corresponding to ice spheres with radii 0.07 and $0.09 \mathrm{~km}$. According to Banfield et al. (1995) the $\mathrm{G}$ and $\mathrm{K}$ events contributed about half of the globally integrated optical depth pro- 
duced by debris particles. The total water mass in the particulate debris would then be in the range 5.6 to $11.2 \times 10^{12} \mathrm{~g}$, corresponding to an ice sphere with radius in the range 0.11 to $0.14 \mathrm{~km}$. The volume fraction of water ice for the globally integrated aerosol debris is then in the range $1 \%$ to $2 \%$ for the range of total aerosol volumes derived by West et al. (1995), and by Banfield et al. Such a low ratio of water to silicate is consistent with the proposal by Zahnle et al. (1995) the impacting bodies were water-poor comet fragments which reached the $\mathrm{NH}_{4} \mathrm{SH}$ cloud level near 2 bars in Jupiter's atmosphere.

Spectral signatures of ammonia vapor, enhanced over the impact sites, were observed in the ultraviolet and infrared (Noll et al. 1995; Orton et al. 1995a). Ammonia would favor the condensed phase at temperatures less than about $140 \mathrm{~K}$, in the pressure range between about $10 \mathrm{mb}$ and $600 \mathrm{mb}$. Ammonia should be considered as a potential aerosol constituent, although no estimate of its volume fraction is possible. Aerosols near the $140 \mathrm{~K}$ temperature level (where $\mathrm{P}=12 \mathrm{mb}$ ) and deeper may provide a source of $\mathrm{NH}_{3}$ gas in the stratosphere lasting many months. Heating by sunlight could produce grain temperatures that are somewhat above the gas temperature at the same location, stimulating $\mathrm{NH}_{3}$ ice sublimation.

Silicate and water ice can account for $10 \%$ to $20 \%$ of the total particle volume, but neither can account for the debris spectra at wavelengths shorter than $2 \mu \mathrm{m}$. Water and ammonia ice do not absorb significantly at wavelengths less than $1 \mu \mathrm{m}$ and must be mixed with some absorbing material. This material absorbs more efficiently at short wavelengths, as indicated in Fig. 4. Graphite and silicate are candidates for the absorbing material, but they have imaginary refractive indices which are too flat compared to the derived values. Carbon disulfide was observed in UV spectra (Noll et al. 1995; Atreya et al. 1995) and appeared to be an attractive candidate for producing the particles in the waves, but it lacks significant absorption at most wavelengths. Elemental sulfur was also observed in UV spectra (Noll et al. 1995). Moses et al. (1995) and Zahnle et, al. (1995) showed that most of the gas-phase sulfur would be converted to $\mathrm{S}_{8}$ which forms a solid. It would therefore be expected to contribute to the aerosol mass. But it too lacks significant absorption at red wavelengths.

The interpretation of the debris spectra may not be as simple as deriving a value for the imaginary part of the refractive index as West et al. (1995) and others have done. If the particles are a heterogeneous mix of two or more components, the use of Mie theory for a homogeneous sphere may not be appropriate. It is likely that very small (a $<0.1 \mu \mathrm{m})$ graphite and silicate grains condensed early in the cooling phase of the plume and served as condensation nuclei for water ice, sulfur and organics (Field et al. 1995; Field, 1995; Friedson, 1995). Graphite has an absorption maximum near $250 \mathrm{~nm}$. It is conceivable that a mixture of very small graphite and silicate grains with larger water ice grains could produce the observed reflectivity, although a quantitative test of that hypothesis has not been done.

The role of carbon in the impact process raises many questions. If all the carbon in the impacting body and in the jovian atmosphere which became entrained in the fireball were processed through a high temperature phase of several thousand $K$, and if oxygen from the comet or Jupiter were sufficiently abundant, all of the carbon would end up as CO which is stable as a gas in the atmosphere. CO was observed, in significant abundance (see the chapter by Lellouch), but observations of CS and $\mathrm{CS}_{2}$, and $\mathrm{HCN}$ indicate that carbon in the form of organic material may be abundant. There is also a rationale for expecting carbon soot to be one of the refractory materials that condenses first in the cooling fireball (Friedson, 1995). The ratio of carbon to oxygen is a key parameter.

The heterogeneous nature of the complex process of fireball formation and evolution, fallback, entrainment and mixing of the comet material and jovian gas, and the chemistry 
of the shock and radiation environments near the eruption leave open the possibility of formation of a variety of constituents under a wide variety and rapidly evolving thermodynamic and chemical states. Field (1995) recently pointed out that refractory grains which form first as the plume cools could catalyze the formation of organics from $\mathrm{CO}$ reacting with $\mathrm{H}_{2}$ through a Fisher-Tropsch process (Anders, 1971), and that water might be bound to silicates as water of crystallization as proposed by Anders for the cooling solar nebula. The relatively low temperature organic condensates and ices (water and possibly ammonia) would condense on previously formed refractory silicate and graphite nuclei and form grain mantles which contribute most to the particle volume.

Wickramasinghe and Wallis (1994) anticipated that submicron particles of organic material would be produced by the impacts. West et al. (1995) noted that the shape of the derived refractive index spectrum was similar to that measured for polymerized hydrogen cyanide (poly-HCN). Wilson and Sagan (1995) showed that the imaginary refractive indices of an organic residue from the Murchison carbonaceous chondrite provide an excellent match to the derived spectrum at wavelengths less than $1 \mu \mathrm{m}$. Noting that several organic gas-phase molecules were observed, including CS, $\mathrm{CS}_{2}$ (Noll et al. 1995), and HCN (Marten et al. 1995), and that some organics are sufficiently volatile to exist in the gas phase as needed to produce particles in waves at temperatures near $150 \mathrm{~K}$, West et al. proposed that an organic material rich in $\mathrm{S}$ and $\mathrm{N}$ is responsible for the color of the particles. A cornerstone of the argument is that the particles produced by the waves are observed to have the same color as the more permanent debris particles (Fig. 2), suggesting that the same material is responsible for both. The only way that could happen is to have the material which produces the color to favor the solid phase at temperatures where the long-lived particles reside (in the vicinity of $1 \mathrm{mb}$ or deeper), while the particles in the waves are at a slightly higher altitude where the ambient temperature is higher and the thermodynamics favor the gas phase before and after the passage of the wave. While simple organics like $\mathrm{CS}_{2}$ are sufficiently volatile to condense near $150 \mathrm{~K}$, more complex organics are usually less volatile. Poly-HCN seems unlikely now that Ortiz et al. (1995) derived a low value for the imaginary refractive index at $1.58 \mu \mathrm{m}$ (see Fig. 4), while $\mathrm{k}$ for poly-HCN remains high at that wavelength. A candidate or mixture with the right thermodynamic and spectral properties remains to be identified.

\section{Particle Vertical Structure}

The impact debris particles appear as bright features in strong methane absorption band images and as dark features in ultraviolet images (Fig. 1). Those contrasts are produced because the particles are higher in the atmosphere than the background aerosol, reflecting photons that would otherwise be absorbed in the methane bands, and absorbing photons that would otherwise be backscattered at ultraviolet wavelengths by $\mathrm{H}_{2}$ and $\mathrm{He}$ molecules. The latitude of the impacts (near $43^{\circ} \mathrm{S}$ ) was fortuitous for their visibility because Jupiter's intrinsic reflectivity in the strong methane bands is lowest at that latitude. The deeper tropospheric ammonia clouds which occupy pressure levels between about 350 and $600 \mathrm{mb}$ at $43^{\circ} \mathrm{S}$ normally account for almost all of the reflected light at that latitude in the strong methane bands. Further to the south, beginning near $60^{\circ} \mathrm{S}$, Jupiter's polar stratospheric haze darkens the planet in the UV and brightens it in the strong methane bands for the same reason the impact particles do. Except for their location closer to the equator, the impact particles are hard to distinguish from the polar haze particles, indicating similar altitudes and optical properties. West et al. (1992) summarized observations regarding the polar haze and argued that they extend upwards to pressure levels near $1 \mathrm{mb}$. 


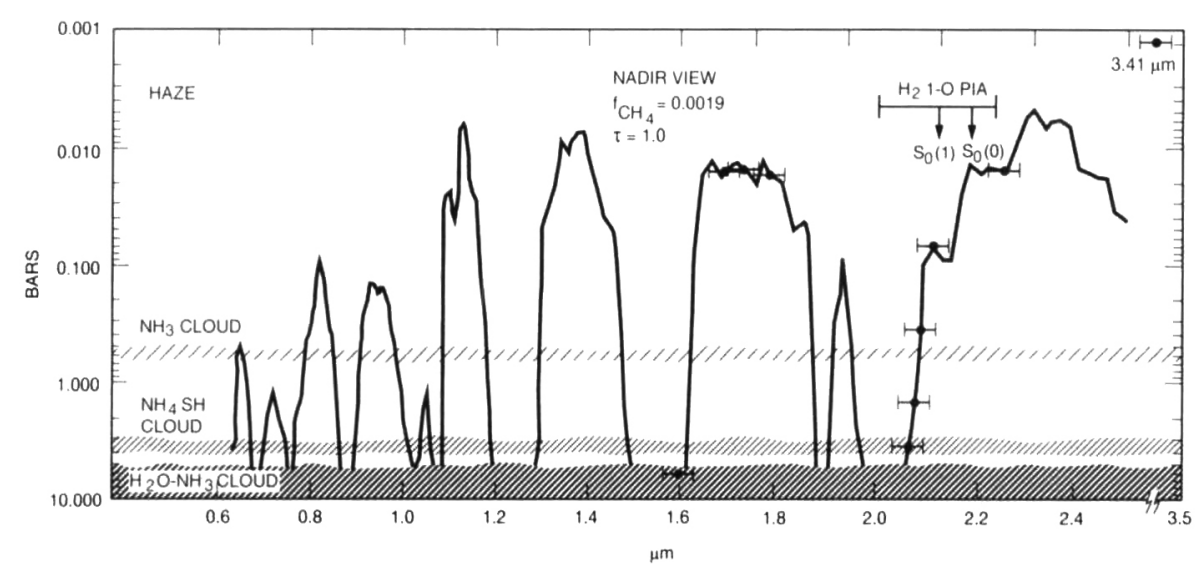

FIGURE 8. The pressure level coresponding to unit optical depth from methane and hydrogen pressure-induced absorption (PIA) are shown at visible and near-IR wavelengths, along with locations of cloud layers in Jupiter's upper troposphere and stratosphere (adapted from Baines et al. 1993). Points with error bars mark locations of filters used by K. H. Baines and others to observe Jupiter at the NASA Infrared Telescope Facility.

One measure of the vertical location in a plane-parallel atmosphere sampled by photons at different wavelengths is the pressure level where unit optical depth is reached for light directed toward the nadir. At that location a fraction 1/e of the upward-directed photons will emerge without being scattered or absorbed. When the solar incidence and viewing directions are away from the zenith and nadir, the equivalent depth of penetration is smaller by the factor $0.5\left(1 / \mu+1 / \mu_{0}\right)$ where $\mu=\cos (\theta), \mu_{0}=\cos \left(\theta_{0}\right)$, and $\theta$ and $\theta_{0}$ are the angles between the surface normal and the view and sun directions, respectively. Unit optical depth for scattering by molecular $\mathrm{H}_{2}$ and He in HST images taken with the F255W filter with effective wavelength $275 \mathrm{~nm}$ (Fig. 1) occurs at 0.35 bar. At other UV wavelengths unit optical depth from gas molecules scales approximately as $\lambda^{-4}$. The wavelength dependence of methane and hydrogen gas determines the level of photon penetration in the near-infrared. Fig. 8 shows that dependence.

Methane absorption coefficients range over more than three orders of magnitude. Photons in the strong band at $3.4 \mu \mathrm{m}$ sample the 1-mb level of the stratosphere. At that wavelength the contrast between belts and zones, and even the Great Red Spot, which is an elevated tropospheric cloud, disappears. Figures 9-11 show Jupiter at selected wavelengths which sample a range of methane absorption coefficients. The appearance of the planet after the impacts (Figs. 10 and 11) can be compared with that before the impacts (Fig. 9).

Images at $3.41 \mu \mathrm{m}$ sample only altitudes higher than the 10 -mb pressure level, and unit optical depth for vertical viewing occurs near the 1-mb level. Prior to the impacts only reflection from the high-latitude haze, and emission from the $\mathrm{H}_{3}^{+}$ion, also at polar latitudes, was seen. Shortly after the impacts reflection from haze particles near latitude $45^{\circ} \mathrm{S}$ is prominent (Fig. 10), proving that impact particles reside at altitudes at least as high as the 10-mb level, and perhaps much higher. Orton et al. (1995b) showed that only a very faint trace of particle scattering could be seen near latitude $45^{\circ} \mathrm{S}$ by 11 October, 1994 , and none could be seen in a 3.4- $\mathrm{m}$ m image taken on 1 February, 1995. From these observations it appears that the e-folding time for particle sedimentation near the 1-mb level is 4-6 weeks, corresponding to the sedimentation time calculated for sphere of radius 0.07-0.1 $\mu \mathrm{m}$ and density $2 \mathrm{~g} \mathrm{~cm}^{-3}$ (West et al. 1995). 


\section{April 28 (UT)}

LCM $=55$ degrees

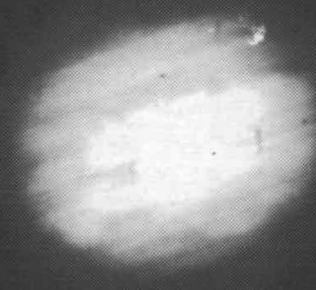

$1.59 \mu m$

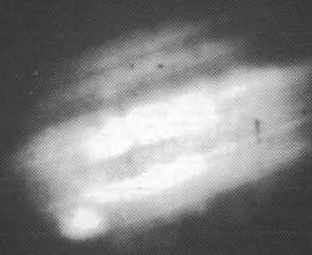

$1.80 \mu \mathrm{m}$

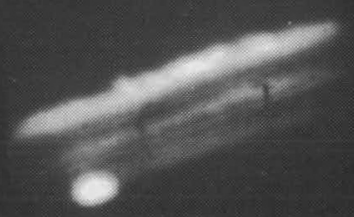

$2.00 \mu \mathrm{m}$

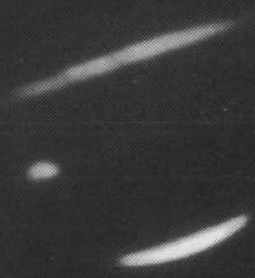

$2.10 \mu \mathrm{m}$

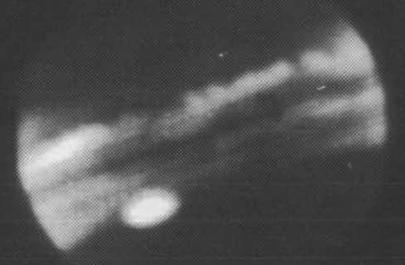

$2.00 \mu \mathrm{m}$
$3.80 \mu \mathrm{m}$

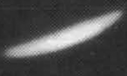

$227 \mu m$

FIGURE 9. Images of Jupiter in the near-infrared methane bands observed with the NASA IRTF telescope (courtesy K. H. Baines and G. Orton. These images were taken three months prior to the comet impacts. The Great Red Spot is the bright (between wavelengths 1.8 and $2.9 \mu \mathrm{m}$ ) oval about half way between the central meridian and the morning limb. 


\section{August 3 (UT) \\ $\mathrm{CML}=63^{\circ}$}

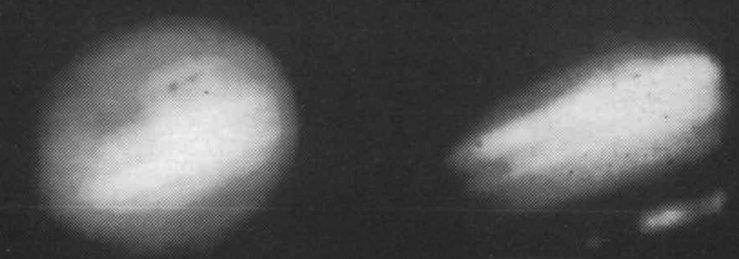

$1.80 \mu \mathrm{m}$

$$
H \rightarrow-=\prod_{G}
$$

$2.10 \mu \mathrm{m}$

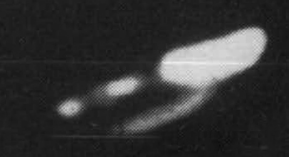

$2.27 \mu m$

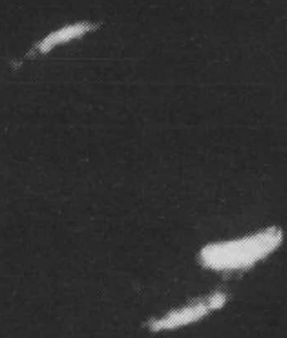

$3.41 \mu m$

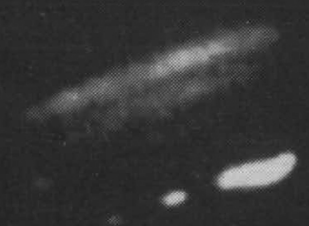

$3.80 \mu \mathrm{m}$

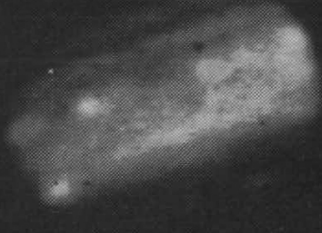

$4.00 \mu \mathrm{m}$

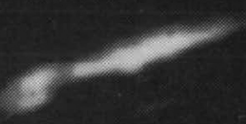

$5.05 \mu m$

FIGURE 10. Images of Jupiter in the near-infrared methane bands observed with the NASA IRTF telescope (courtesy K. H. Baines and G. Orton, and the IRTF SL9 otserving team-see Orton et al. 1995a). These images were taken about 10 days after the last impact. Several of the impact sites are identified on the $2.10-\mu \mathrm{m}$ image. 


\section{April 06 (UT)}

LCM $=162$ desrees

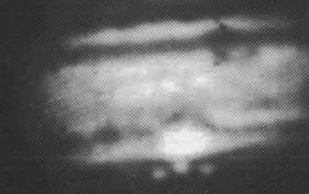

$1.85 \mu \mathrm{m}$

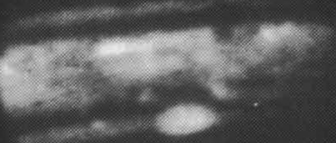

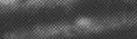

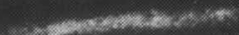

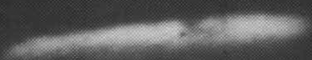

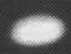

$2.00 \mu \mathrm{m}$

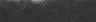

-

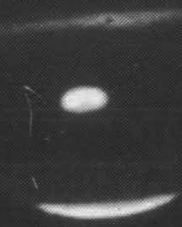

$2.10 \mu \mathrm{m}$

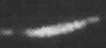

$3.30 \mu \mathrm{m}$

$3.58 \mu \mathrm{m}$

FIGURE 11. Images of Jupiter in the near-infrared methane bands observed with the NASA IRTF telescope (courtesy G. Orton and K. H. Baines). These images were taken almost 9 months after the impacts and show the remants of the impact-generated aerosols at 2.1 and $2.27 \mu \mathrm{m}$. 
Evidence for particles at higher altitudes comes from measurements of satellite eclipses. Mallama et al. (1995) infer the presence of impact particles at altitudes as high as $300 \mathrm{~km}$ above the 1-bar level one month following the impacts. At that altitude the pressure is a few $\mu \mathrm{b}$. The vertical optical depth of these particles is only 0.1 at $540 \mathrm{~nm}$ wavelength and much less at 2-4 $\mu \mathrm{m}$, assuming the extinction coefficient of 0.001 derived by Mallama et al. for the top of the haze layer applies to a $100-\mathrm{km}$ thick layer. If the particles were formed during the emplacement of the fallback material they would have to be much smaller than $0.1 \mu \mathrm{m}$ radius, since particles of radius $0.1 \mu \mathrm{m}$ sediment out in a matter of hours at such a low pressure. Alternatively, gas-phase material at a few $\mu \mathrm{b}$ pressure may be a source of solid particles one month after the impact. Moses et al. (1995) and Zahnle et al. (1995) pointed out that $\mathrm{S}_{2}$ gas will be converted to solid $\mathrm{S}_{8}$ although the time scale for that process should be much shorter than one month.

Banfield et al. (1995) took advantage of the the broad range in depth probed by different wavelengths in the $2-\mu \mathrm{m}$ methane spectrum to derive haze vertical structure and optical depth in late July, mid-August, and late August of 1994. Their inversion algorithm always selected the highest altitude and lowest pressure (20 mb or less) as the location of the haze.

West et al. (1995) examined the darkest cores of several fresh impact sites using WFPC2 images at UV $(275 \mathrm{~nm})$ and in the $893-\mathrm{nm}$ methane band. They found that the particles need to extend up to pressures of a few mb or less to produce the low reflected UV intensity at the limb. But material confined to pressures less than about $100 \mathrm{mb}$ produced a center-to-limb behavior in the methane band that was not consistent with observations. The observations required that a significant optical depth of particles extending to pressure levels as high as $200 \mathrm{mb}$. Baines et al. (1995) and Orton et al. (1995a) found that such a model was consistent with reflectivity in the near-infrared methane bands. Baines et al. derived particle column number density of $3 \times 10^{8} \mathrm{~cm}^{-2}$ for core of the G site on 3-August-1994. Models with aerosols extending down to $400 \mathrm{mb}$ constructed by both West et al. and Moreno et al. (1995) were able to fit HST and ground-based data.

Vertical structure models point to two types or regions formed from the impacts. In dense cores the particles extend over many scale heights, from $200 \mathrm{mb}$ pressure or deeper to a few mb pressure or less. In the diffuse outlying regions the particles cover a much larger areal extent but their optical depth is smaller than in the cores and they are confined to altitudes higher than the $20-\mathrm{mb}$ level. This picture is consistent with the idea that particles in the outlying regions were emplaced from above by fallback from the plume, but that particles in the core region were emplaced both from above and below by plume fallback and by turbulent entrainment and buoyancy of the hot rising fireball.

\section{Particle Microphysical Processes and Evolution}

Observations of plumes shortly after impact, and observations of ultraviolet, visible, and infrared emissions by hot plume material (see the chapters in this volume by Hammel, Chapman, and Nicholson) have confirmed and enlarged upon many aspects of models of the events surrounding the impacts (see chapters by Crawford, MacLow and Zahnle). The main features in common to all the models of the largest impacts include an incoming bolide which creates a shock wave and an eruptive plume which cools from several thousand $\mathrm{K}$ to much lower temperature as it expands and travels back along the incoming path to altitudes near $3000 \mathrm{~km}$, followed by fallback into the upper atmosphere and heating upon re-entry. The process is heterogeneous, with temperature, pressure and composition all functions of location and time. Models differ in terms of the depth of 
penetration attained, and the fractions of the impacting body and Jupiter gas which was ejected in the plume, and to what distances from the impact site various parcels came to rest. How did grains form during this process, and what may have been their size distribution and composition as functions of time?

Field et al. (1995) and West et al. (1995) were the first to examine the possible compositions and microphysical processes which shaped the grain properties during the early stages of the formation of the haze clouds. Both studies pointed out that silicates are expected to form the early condensate if cometary material was present, and observations by Nicholson et al. (1995) confirmed that premise.

The most recent and thorough work to date was done by Friedson (1995) who looked at two possible compositions for the hot initial fireball and studied the formation of $\mathrm{Al}_{2} \mathrm{O}_{3}$, silicate, and soot grains. Friedson's work builds on previous models of grain formation in stellar atmospheres. The following remarks are based on his work.

The ratio of carbon to oxygen determines whether $\mathrm{Al}_{2} \mathrm{O}_{3}$ or soot will form the refractory grains which condense first from the hot vapor. If $\mathrm{Al}_{2} \mathrm{O}_{3}$ grains appear they will serve as condensation nuclei for less refractory materials like metallic iron, and magnesium and iron silicates. Friedson argued that the environment within each fireball may have been sufficiently heterogeneous that both types of grains formed in different locations. SiC or $\mathrm{SiS}$ may also have been abundant if the ratios of carbon or sulfur to silicon atoms were favorable.

The most refractory grains should form by homogeneous nucleation from the vapor phase. The process is complex because the free energy of the small atomic clusters are thought to dominate the nucleation process, and the formalism surrounding homogeneous nucleation is based on a free energy which is not easily calculated for such small particles. Formation of soot is further complicated by the empirical finding from flame studies that small soot particles tend to agglomerate to larger sizes rather than by direct growth of particles from the vapor. The chemistry of soot formation is complex, beginning with $\mathrm{C}_{2} \mathrm{H}_{2}$ reacting with aromatic hydrocarbons in such a way that the $\mathrm{C} / \mathrm{H}$ ratio is enhanced as the molecular weight increases. Many organic molecules, including polycyclic aromatic hydrocarbons, and even fullerenes may be important in grain formation.

The main events governing particle microphysics as described by Friedson are shown in Figure 12. The process of mantling of $\mathrm{Al}_{2} \mathrm{O}_{3}$ by magnesium and iron silicates was omitted from the figure because it occurs very shortly after formation of the $\mathrm{Al}_{2} \mathrm{O}_{3}$ grains. The pressure/temperature model used in the calculation was taken from Zahnle et al. (1995). The temperature at $20 \mathrm{~s}$ after impact is $3400 \mathrm{~K}$. Grain formation occurs when $\mathrm{T} \sim 2000 \mathrm{~K}$. Mantling by more volatile species (organics and ice) occurs at much lower temperature. Volatiles which may condense during the initial cooling phase of the plume are expected to vaporize upon re-entry into the atmosphere, and then re-condense after the parcels cool to near-ambient temperature.

During the hours to months following the impacts the particles may undergo additional growth by coagulation or from gas to particle conversion, and their numbers in the stratosphere will decrease by sedimentation to the troposphere where rapid vertical mixing will remove them. Time scales for sedimentation and coagulation as a function of pressure are shown in Fig. 13. West et al. (1995) noted that the increase in particle mean radius during the month following the impacts (from 0.21 to $0.28 \mu \mathrm{m}$ ) was consistent with the idea that particle coagulation was taking place in the dense core regions. West et al. and Banfield et al. (1995) reported a decrease in total particle volume after one month, indicating sedimentation is also taking place.

The disappearance of particles from the few-mb level or higher as seen in the 3.4- $\mu \mathrm{m}$ methane band images two months after impact (Orton et, al. 1995b) is consistent with 


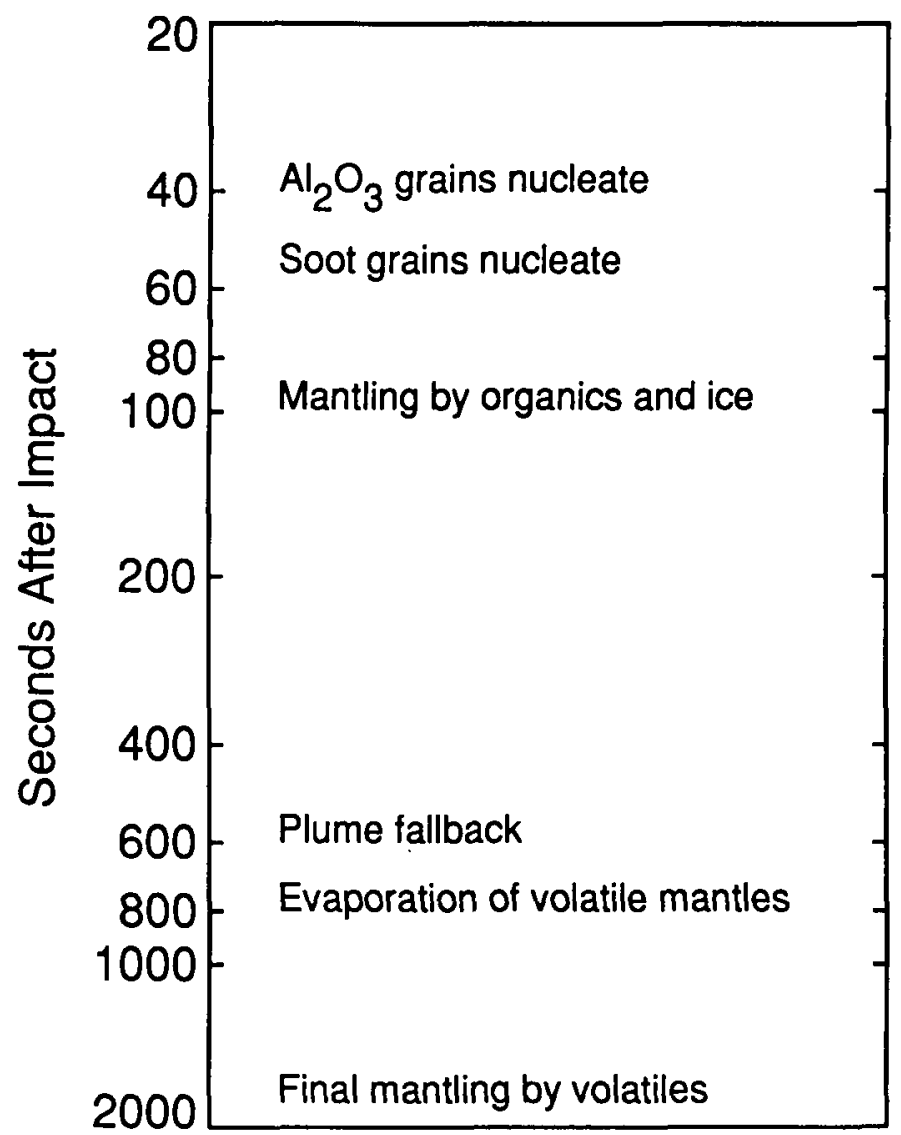

FIGURE 12. Particle microphysical processes during the first $2000 \mathrm{~s}$ in an evolving fireball/plume as discussed by Friedson (1995).

particle radius greater than about $0.1 \mu \mathrm{m}$. Few particles with radii near $0.3 \mu \mathrm{m}$ or larger should reside at pressure levels less than $100 \mathrm{mb}$ after one year. Deeper in the atmosphere vertical mixing from the troposphere increases rapidly, removing particles. Haze particles can be seen in images taken about a year after the impacts (see Fig. 11), although their number density is clearly much lower than it was a month after impact. Part of the decrease in surface density, perhaps a factor of $2-3$ is due to horizontal spreading, but a significant part is due to sedimentation. The haze is most visible in the images at 2.1 and $2.27 \mu \mathrm{m}$ which sample pressures in the range 10 to $100 \mathrm{mb}$. The most recent images show particles reaching latitudes near $20^{\circ} \mathrm{S}$. It is difficult to tell how far toward the south pole the particles may have reached because of the strong gradient in Jupiter's polar stratospheric haze. As the particles continue to disperse they will tell us much about meridional transport in the jovian stratosphere.

\section{Summary}

After initial analysis of some of the voluminous data obtained on particles created by the SL9 impacts, a consensus view is emerging regarding the broad questions of particle origin, composition, and evolution. Cometary material is responsible for a significant portion of the particle total volume, although silicate material accounts for only $10-20 \%$ 


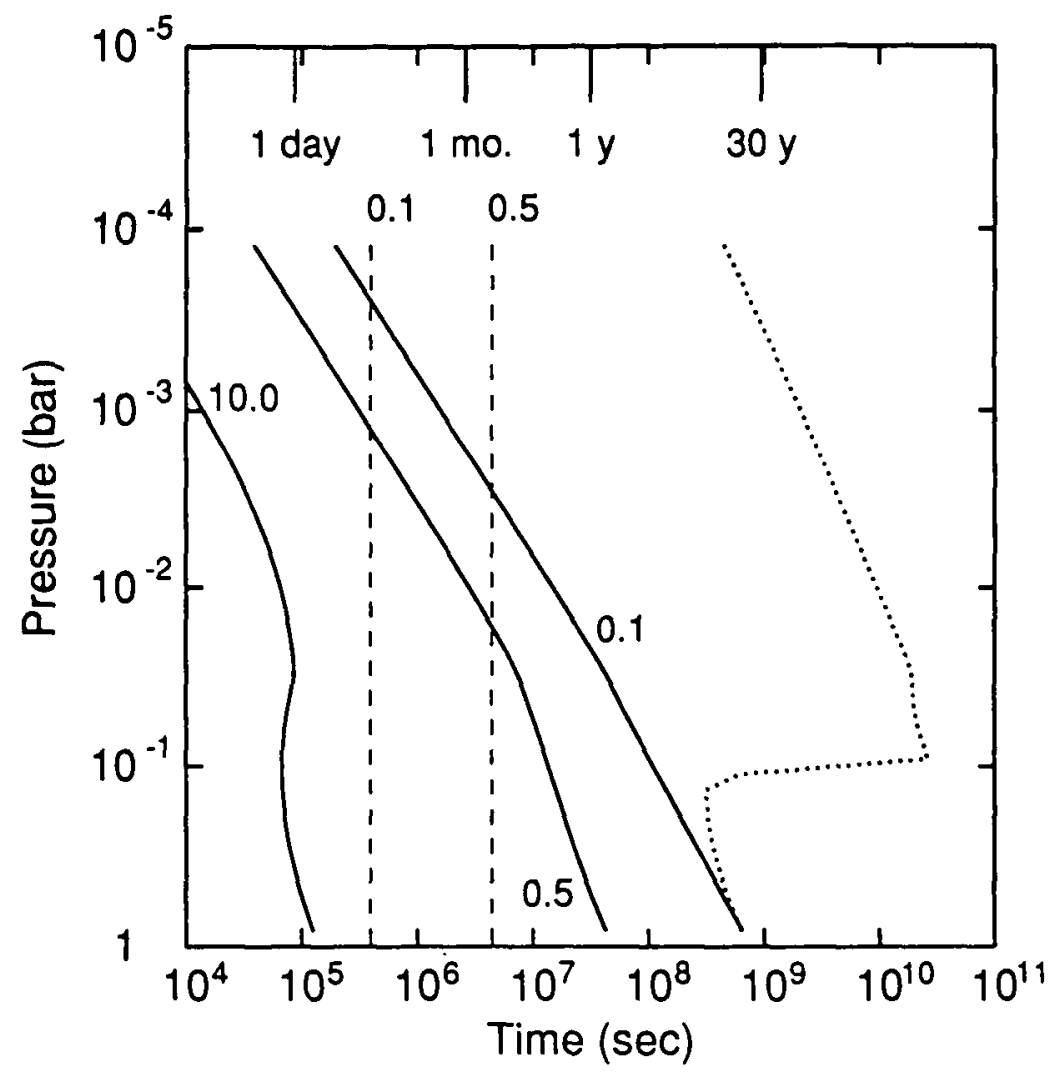

FIgURE 13. Time scales for sedimentation and coagulation of impact particles are shown as a function of pressure and particle mean radius (assuming spherical shape) from West et al. (1995). Solid curves indicate sedimentation times for particles of the indicated radius. Dashed curves indicate coagulation times for an initial population of 0.1 and $0.5 \mu \mathrm{m}$ particles. The initial particle number density for the two cases was taken to be 570 and $23 \mathrm{~cm}^{-3}$, respectively, consistent with particle optical depths determined for dense core regions by West et al. The dotted curve indicates the eddy diffusive time scale.

of the particle volume. The plumes on the limb were made visible by refractory grains which include silicates but may also contain aluminum oxide and soot. Onto these grains condensed more volatile material, including a small amount for water ice and probably organics, with ammonia possibly contributing as well. The color of the particles and their ability to form during the passage of a stratospheric wave suggest some kind of volatile organic material, but a specific candidate with both the spectral and thermodynamic properties has not been identified.

The total volume of particles (equal to that of a sphere with diameter approximately $1 \mathrm{~km}$ ) is consistent with expectations of many of the hypervelocity impact models. The particles behave optically and dynamically like spheres with radii in the range 0.1 to $0.3 \mu \mathrm{m}$, although they may be aggregates of smaller spheres. Their color is homogeneous on a global scale, a puzzling fact since many heterogeneous processes were operative over a wide range of distance and time scales during the impact, rebound, and fallback phases of the events. This heterogeneity may be reflected in the internal heterogeneity of particle composition, but we have no observations which bear on that question.

Most of the particles at locations distant from the dark core regions were emplaced near the $1 \mathrm{mb}$ pressure level, with an uncertainty of a factor of about 3 , constrained 
by reflectivity in UV and near-IR wavelengths and by the sedimentation time scale for a $0.25-\mu \mathrm{m}$ radius particle. The report of particles at much higher altitudes implies a source (gas-to-particle conversion) and/or the particles at very high altitudes must have radii much smaller than $0.1 \mu \mathrm{m}$. Particles within the dark core regions extend over a large altitude and pressure range, from a few $\mathrm{mb}$ to $200 \mathrm{mb}$ or deeper. Such a distribution probably reflects the different emplacement mechanisms. Particles distant from the cores were part of plume fallback, while particles near the center could come from entrainment of upward flowing gas near the shock tube, thermal upwelling, as well as fallback. As the debris pattern continues to spread in latitude over the course of many months, it is behaving much like volcanically-produced sulfuric acid particles in the earth's stratosphere and is providing new and unique information on transport in the jovian stratosphere.

\section{REFERENCES}

Anders, E. 1971 Meteorites and the early solar system. Ann. Rev. Astron. Astrophys. 9, (eds. L. Goldberg, D. Layzer, \& J. Phillips), pp. 1-34. Ann. Reviews, Inc.

atreya S. K., Edgington S G., Trafton L. M., Caldwell J. J., Noll K. S., \& Weaver, H. A. 1995 Abundances of ammonia and carbon-disulfide in the jovian stratosphere following the impact of comet Shoemaker Levy 9. Geophys. Res. Lett. 22, 1625-1628.

Baines, K. H., West, R. A., Giver, L. P., \& Moreno, F. 1993 Quasi-random narrowband model fits to near-infrared low-temperature laboratory methane spectra and derived exponential-sum absorption coefficients. J. Geophys. Res. 98, 5517-5529.

BAINEs, K. H., et al. 1994 The effect of SL9 on Jupiter's vertical aerosol structure: Results from IRTF near-infrared imaging Bull. Amer. Astron. Soc. 26, 1591.

Banfield, D., Gierasch, P., Squyres, S., Nicholson, P., Conrath, B., \& Matthews, K. $19952 \mu \mathrm{m}$ spectrophotometry of jovian stratospheric aerosols-scattering opacities, vertical distributions and wind speeds. submitted for publication (Icarus).

Bjoraker, D., Stolovy, S. R., Herter, T. L., Gull, G. E., \& Pirger, B. E. 1995 Detection of water after the collision of fragments $\mathrm{G}$ and $\mathrm{K}$ of comet Shoemaker-Levy 9 with Jupiter. submitted for publication (Icarus).

CLARKE, J., et al. 1995 HST far-ultraviolet imaging of Jupiter during the impacts of comet Shoemaker-Levy 9. Science 267, 1302-1307.

Conrath, B. J., Gierasch, P. J., \& Leroy, S. S. 1990 Temperature and circulation in the stratosphere of the outer planets. Icarus 83, 255-281.

Tabulated optical Properties of graphite and silicate grains 1985 Astrophys. $J$. Supp. Ser. 57, 587-594.

FIELD, G. B. 1995 Dust at the SL9 impact sites. In Abstracts for IAU Colloquium 156: The collision of Comet P/Shoemaker-Levy 9 and Jupiter.

Field, G. B., Tozzi, G. P., \& Stanga, R. M. 1995 Dust as the cause of spots on Jupiter. Astron. and Astropys. Lett. 294, L53-L55.

FRIEDSON, A. J. 1995 Refractory grain formation in Shoemaker-Levy 9 fireballs. submitted for publication (Icarus).

Gierasch, P. J., B. J., \& Magalhães, J. A. 1986 Zonal mean properties of Jupiter's upper troposphere from Voyager infrared observations. Icarus 67, 456-483.

Gustavsen, R. L. 1986 Ph.D. Thesis, Washington State University

HAMMEL, H. B., et al. 1995 HST Imaging of atmospheric phenomena created by the impact of comet Shoemaker-Levy 9. Science 267, 1288-1296.

Ingersoll, A. P. \& Kanamori, H. 1995 Waves from the collisions of comet Shoemaker-Levy 9 with Jupiter. Nature 374, 706-708. 
Khare, G. N., Sagan, C., Reid, Thompson, W. R., Arakawa, E. T., Meisse, C. \& TuMINELLO, P. S. 1994 Optical properties of poly-HCN and their astronomical applications. Canadian J. Chem. 72, 678-694.

Mallama, A., Nelson, P., \& Park, J. 1995 Detection of very high altitude fall-out from the comet Shoemaker-Levy 9 explosions in Jupiter's atmosphere. J. Geophys. Res. 100, $16,879-16,884$.

MARTen A., et al. 1995 The collision of comet Shoemaker-Levy 9 with Jupiter: Detection and evolution of HCN in the stratosphere of the planet. Geophys. Res. Lett. 22, 1589-1592.

Meadows, V. \& CRISP, D. 1995 Impact plume composition from near-infrared spectroscopy. In Proceedings of the European SL-9/Jupiter Workshop (eds. R. West and H. Böhnhardt), pp. 239-244. European Southern Observatory.

Moreno, F., Muñoz, O., Molina, A., López-Moreno, J. J., Ortiz J. L., Rodríguez, J., López-JimÉnez, A., Girela, F., LARSon, S. M., \& CAMPINS, H. 1995 Physical properties of the aerosol debris generated by the impact of fragment-H of comet-P Shoemaker-Levy 9 on Jupiter. Geophys. Res. Lett. 22, 1609-1612.

Moses, J. L., Allen, M., \& Gladstone, G. R. 1995 Post-Sl9 sulfur photochemistry on Jupiter. Geophys. Res. Lett. 22, 1597-1600.

Nicholson, P. D., Gierasch, P. J., Hayward, T. L., Mcghee, C. A., Moersch, J. E., Squyres, S. W., Van Cleve, J., Matthews, K., Neugebauer, G., Shupe, D., WeinBerger, A., Miles, J. W., \& ConRath, B. J. 1995 Palomar observations of the R impact of comet Shoemaker Levy 9. 2. Spectra. Geophys. Res. Lett. 22, 1617-1620.

Noll, K. S., Mcgrath, M. A., Trafton, L. M., Atreya, S. K., Caldwell, J. J., Weaver, H. A., Yelle, R. V., BARnet, C., \& EDGINGTON, S. 1995 HST spectroscopic observations of Jupiter after the collision of comet Shoemaker-Levy 9. Science 267, 1307-1313.

Ortiz, J. L., Muñoz, O., Moreno, F., Molina, A., Herbest, T. M., Birkle, K., Böhnhar.DT, H., \& Hamilton, D. P. 1995 Models of the SL-9 collision-generated hazes. Geophys. Res. Lett. 22, 1605-1608.

Orton, G. et al. 1995a Collision of comet Shoemaker Levy 9 with Jupiter observed by the NASA Infrared Telescope Facility. Science 267, 1277-1282.

OrTon, G. et al. 1995b Some Results from the NASA Infrared Telescope Facility ShoemakerLevy 9 observing campaign. In Proceedings of the European SL-9/Jupiter Workshop (eds. R. West and H. Böhnhardt), pp. 123-128. European Southern Observatory.

Roosserote, M., Barucci, A., Crovisier, J., Drossart, P., Fulchignoni, M., LECACHEUX, J., \& Roques, F. 1995 Metallic emission lines during the impacts $L$ and $\mathrm{Q}_{1}$ of comet $\mathrm{P}$ Shoemaker Levy 9 in Jupiter. Geophys. Res. Lett. 22, 1621-1624.

Rosenquist, J, Biraud, Y. G., Cuisenier., M., Marten, A., Hidayat, T., Chountonov, G., Moreau, D., Muller, C., Maslov, I., Ackerman, M., Balega, Y., \& Korablev, O. 1995 Four micron infrared observations of the comet Shoemaker-Levy 9 collision with Jupiter at the Zelenchuk Observatory: Spectral evidence for a stratospheric haze and determination of its physical properties. Geophys. Res. Lett. 22, 1585-1588.

Sasson, R., Wright, R., Arakawa, E. T., Khare, B. N. \& Sagan, C. 1985 Optical properties of solid and liquid sulfur at visible and infrared wavelengths. Icarus 64, 368-374.

WEST, R. A. 1991 Optical properties of aggregate particles whose outer diameter is comparable to the wavelength. Appl. Optics 30, 5316-5324.

West, R. A. \& SMITH, P. H. 1991 Evidence for aggregate particles in the atmospheres of Titan and Jupiter. Icarus 90, 330-333.

West, R. A., Friedson, A. J., \& Appleby, J. F. 1992 Jovian large-scale stratospheric circulation. Icarus 100, 245-259.

West, R. A., Karkoschka, E., Friedson, A. J., Seymour, M., Baines, K. H., \& Hammel, H. B. 1995 Impact debris particles in Jupiter stratosphere. Science 267, 1296-1301.

Wickramasinghe, N. C. \& Wallis, M. K. 1994 Submicron dust and the collision of comet SL-9 with Jupiter. Astrophys. and Space Sci. 219, 295-301. 
Wilson, P. D., \& SAgan, C. 1995 Chemistry of the Shoemaker-Levy 9 jovian impact blemishes: Indigenous cometary vs. shock-synthesized organic matter. In The collision of comet P/Shoemaker-Levy 9 and Jupiter (eds. K. Noll et al.) Cambridge.

Zahnle, K., Maclow, M. M., Lodders, K., \& Fegley, B. 1995 Sulfur chemistry in the wake of comet Shoemaker Levy 9. Geophys. Res. Lett. 22, 1593-1596. 